\title{
Rotamers and Migration: Investigating the Dissociative Photoionization of
}

\section{Ethylenediamine}

Giel Muller, ${ }^{\dagger}$ Krisztina Voronova, ${ }^{\ddagger}$ Bálint Sztáray, ${ }^{{ }^{*}}$ and Giovanni Meloni, ${ }^{\dagger}{ }^{\dagger}$

†University of San Francisco, California USA 94117,

University of the Pacific, Stockton, California USA 95211,

*Corresponding Authors: bsztáray@pacific.edu, gmeloni@usfca.edu
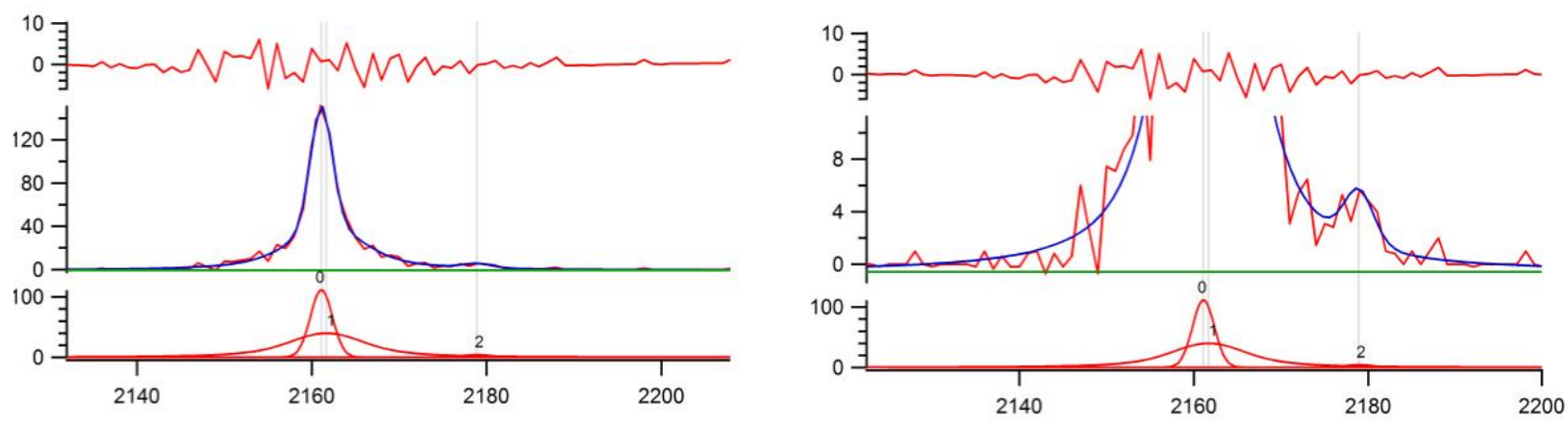

Figure S1. The IGOR multi-peak fitting in the determination of isotopologue contributions to adjacent peaks. Shown above is parent ion $\mathrm{m} / z=60$ and the $\sim 2.3 \%{ }^{13} \mathrm{C}$ and ${ }^{15} \mathrm{~N}$ isotopologue contribution at $8.8 \mathrm{eV}$. 


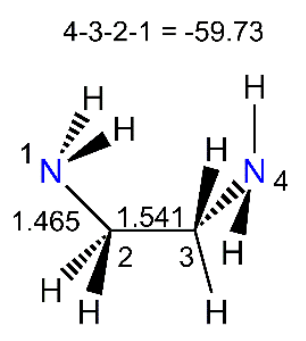

(a) -190.162753

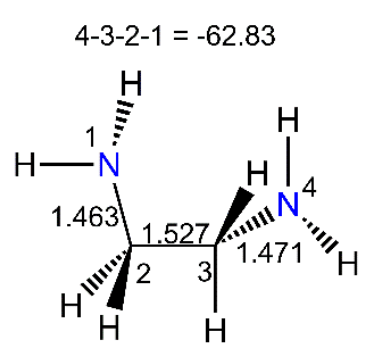

(e) -190.164485

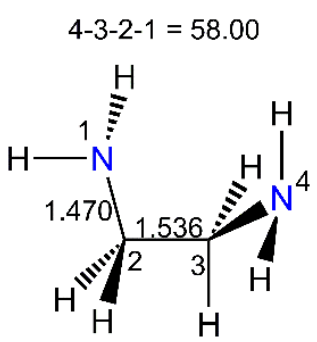

(b) -190.164165

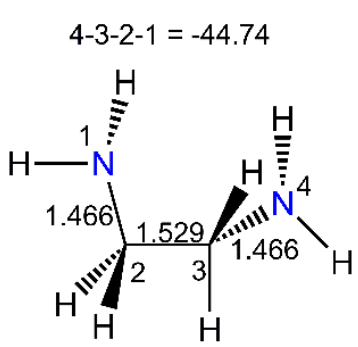

(f) -190.159712

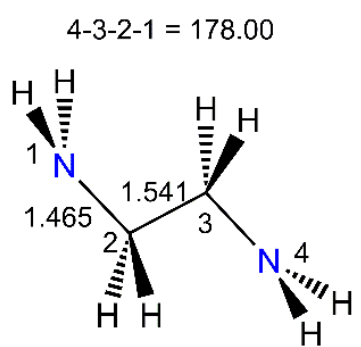

(c) -190.162804

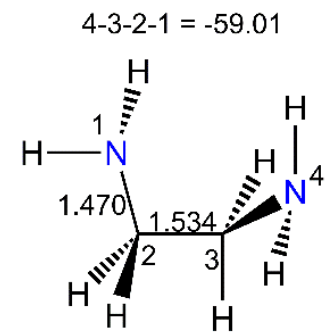

(d) -190.163003

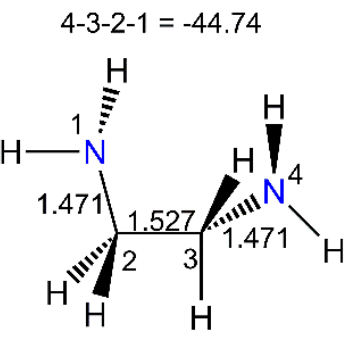

(f) -190.159712
$4-3-2-1=178.00$

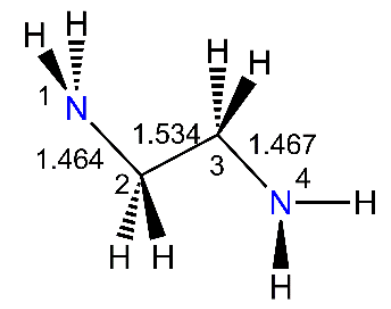

(c) -190.162527

Figure S2. Several conformations of neutral ethylenediamine optimized by CBS-QB3 and energies reported in Hartrees. 


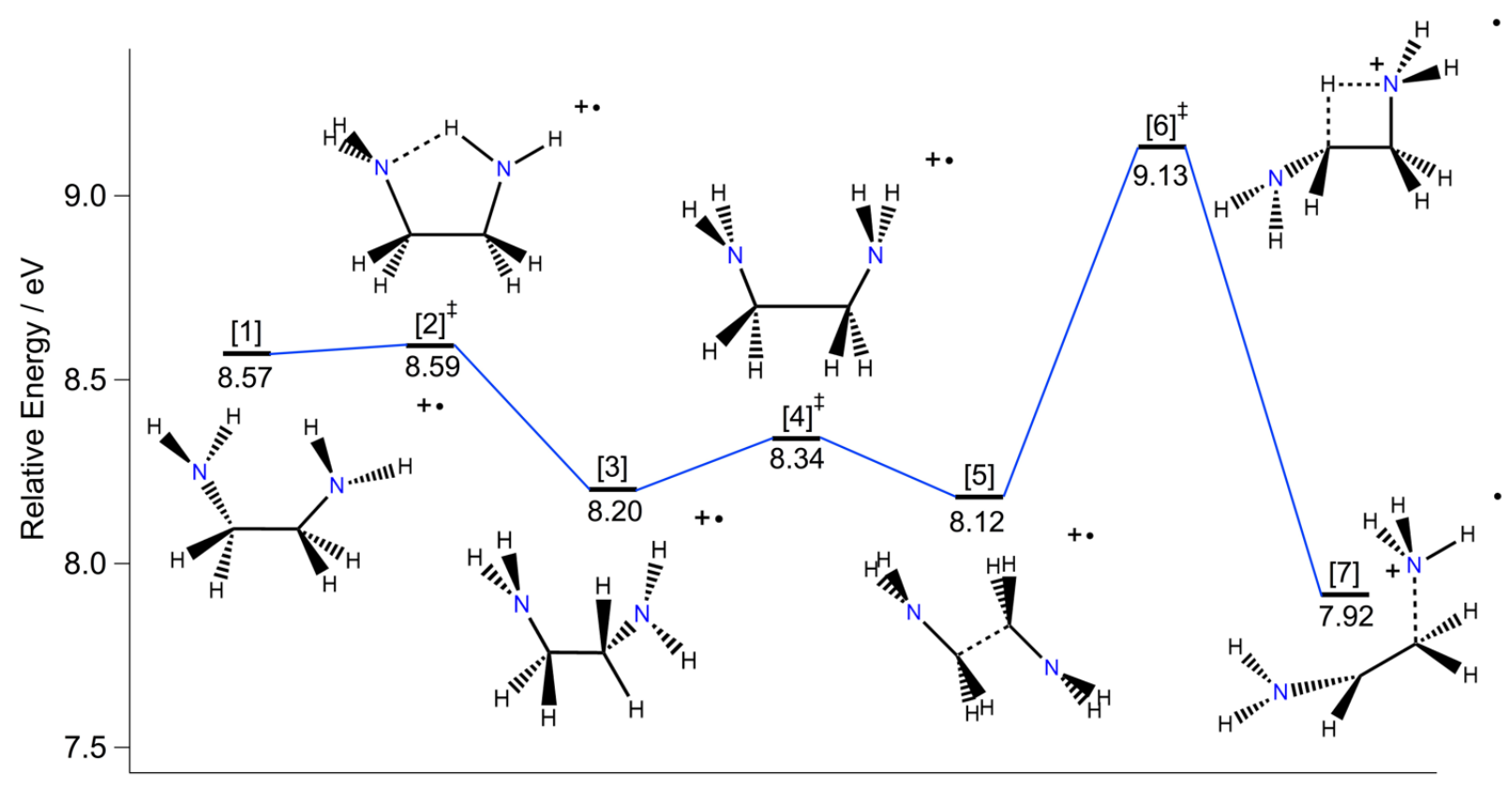

Figure S3. The CBS-QB3 calculated potential energy surface diagram at $0 \mathrm{~K}$ of the initial rotations and H-migrations of ethylenediamine ion. The zero of relative energy is that of the EN neutral. 


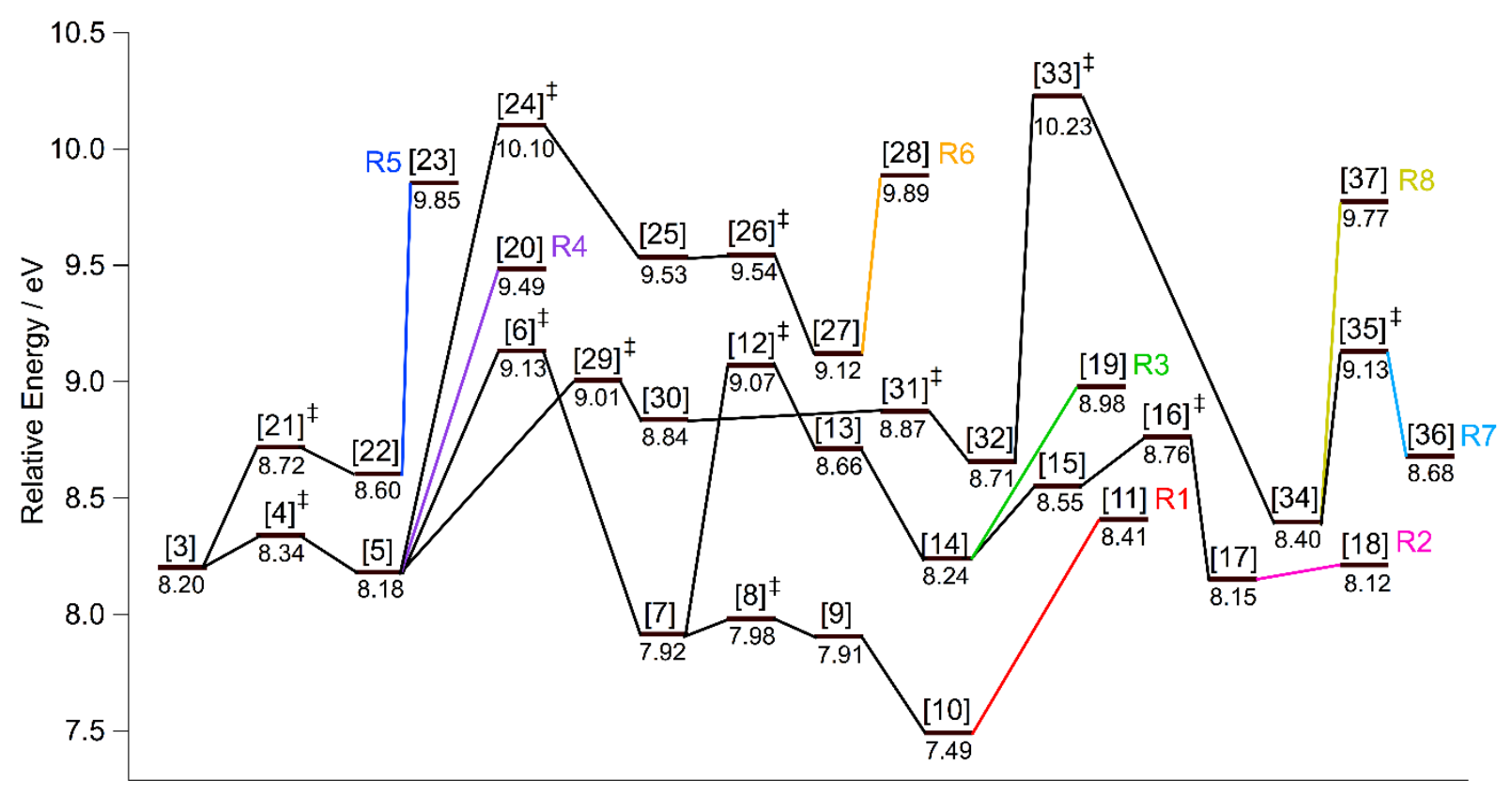

Figure S4. CBS-QB3 calculated potential energy surfaces for the photodissociation channels R1$\mathbf{R 8}$ of the ethylenediamine cation with the relative energies at $0 \mathrm{~K}$ for the stationary points (for structures of the ions, intermediates and TSs see Figure S5). 
[1]

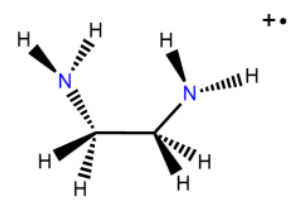

$-189.849513$

[4]

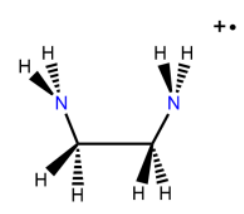

$-189.857991$

[7]

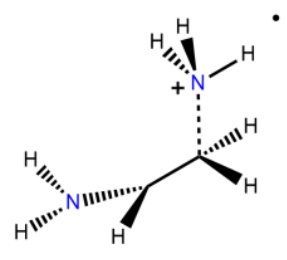

$-189.873607$

[10]<smiles>[1H][CH+][NH+]=CC=C</smiles>

$-189.889117$

[13]

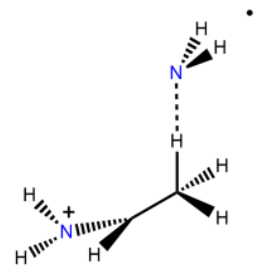

$-189.846509$
[2]

[3]

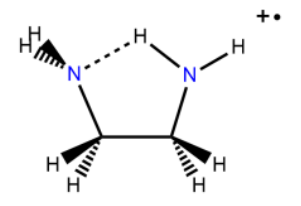

$-189.848718$

[5]

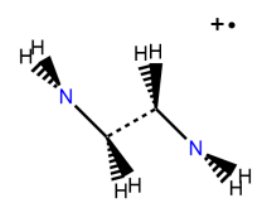

$-189.863825$

$[8]^{\ddagger}$

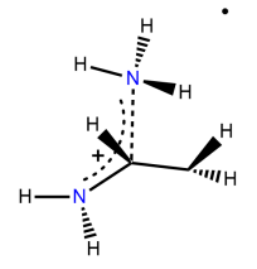

$-189.871216$

[11]

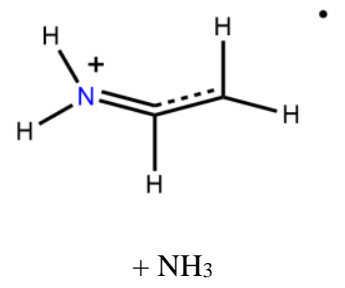

$-133.395330 /-56.460185$

[14]

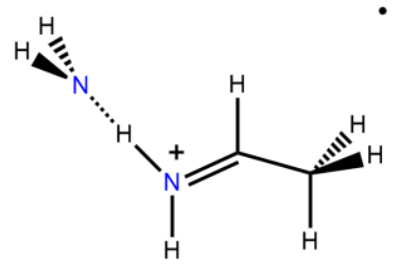

$-189.861845$

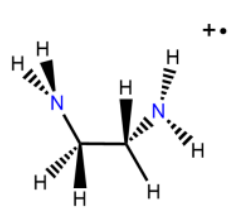

$-189.863091$

[6]

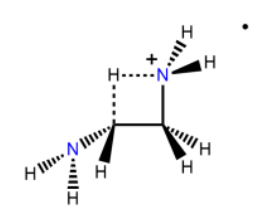

$-189.828841$

[9]

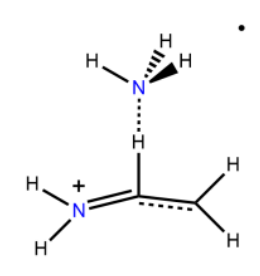

$-189.873965$

[12]

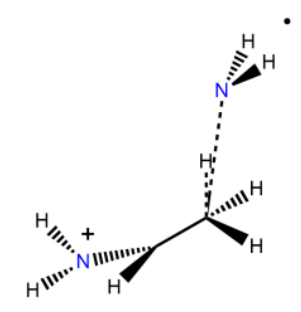

$-189.831115$

[15]

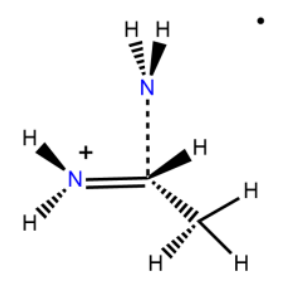

$-189.850212$ 
$[16]^{\ddagger}$

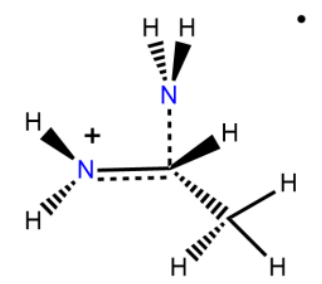

$-189.842500$

[19]

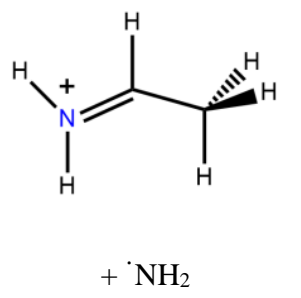

$-134.043361 /-55.791194$

[22]

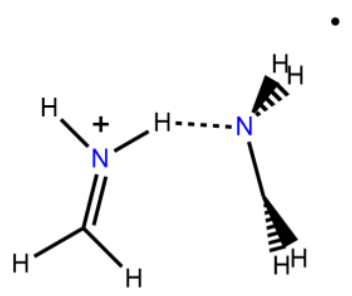

$-189.848278$

[25]

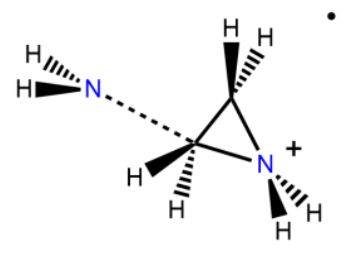

$-189.814095$
[17]

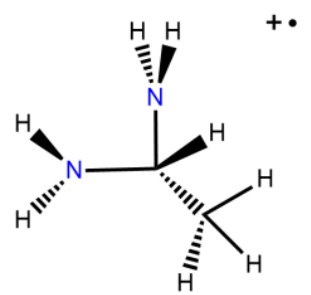

$-189.864917$

[20]
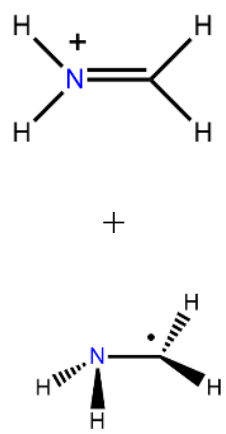

-94.793759 / -95.022133

[23]

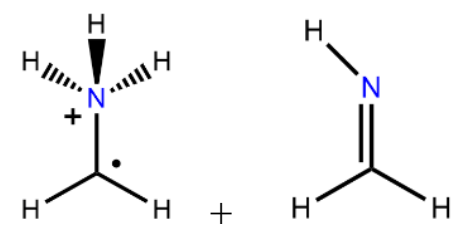

95.337078 / -94.465271

$[26]^{\ddagger}$

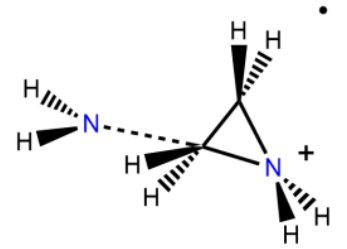

$-89.813774$
[18]

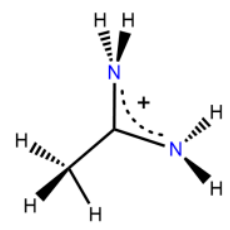

$+{ }^{\circ} \mathrm{H}$

-189.362926 / - -0.499818

$[21]^{*}$

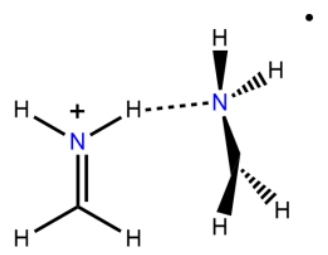

$-189.844050$

$[24]^{\ddagger}$

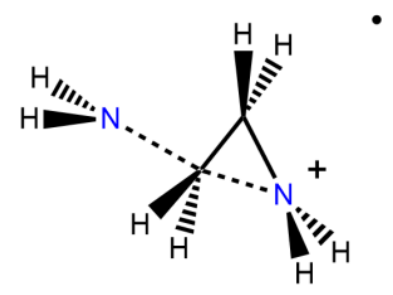

$-189.793238$

[27]

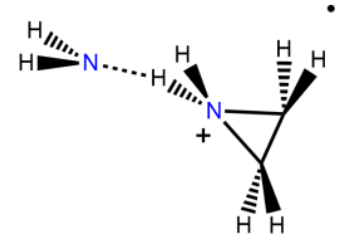

$-189.829306$ 
[28]

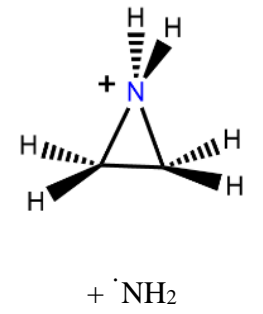

-134.009964 / -55.791194

$[31]^{\ddagger}$

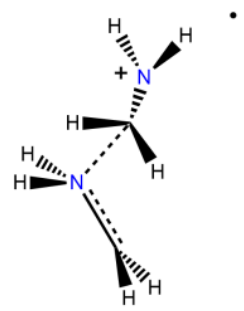

$-189.838413$

[34]

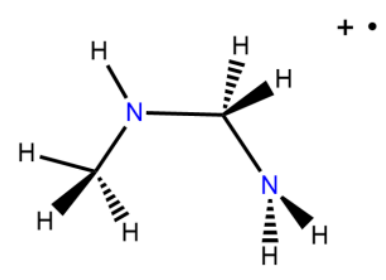

$-189.855912$

[37]<smiles>[1H]C=[N+]([2H])C</smiles>

-94.793759 / -95.011545 $[29]^{\ddagger}$

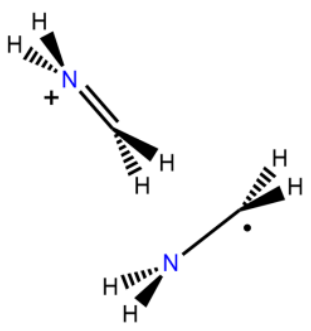

$-189.833513$

[32]

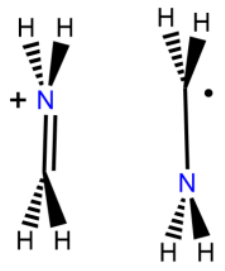

$-189.844059$

[35]

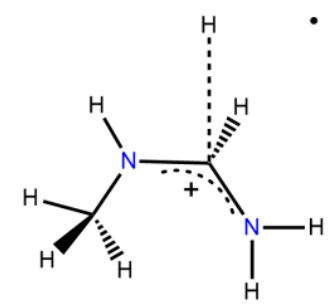

$-189.829040$
[30]

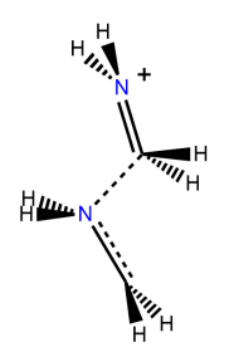

$-189.839696$

$[33]^{\ddagger}$

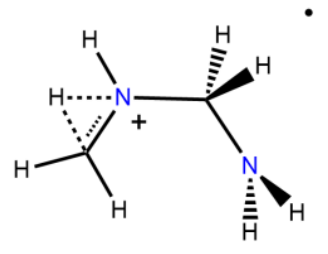

$-189.788605$

[36]

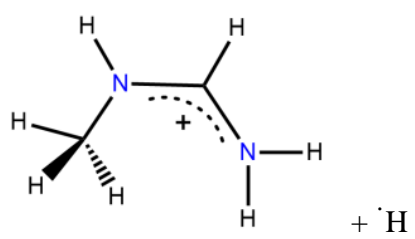

-189.345759 / -0.49982 
Table S1. Geometries for neutral EN and neutral fragments corresponding to stationary points calculated in this work. All calculations were conducted at the CBS-QB3 level.

$\begin{array}{lllll}\text { Neutral EN } & \text { N } & -1.41260800 & -0.59613000 & 0.13611600 \\ \text { H } & -2.24400000 & -0.73360600 & -0.42780700 \\ \text { H } & -1.72345500 & -0.49105600 & 1.09742300 \\ \text { C } & -0.68186300 & 0.60531300 & -0.29660600 \\ \text { H } & -0.60044300 & 0.57313200 & -1.38665400 \\ \text { H } & -1.18004900 & 1.54906400 & -0.02585400 \\ \text { C } & 0.73030700 & 0.59120800 & 0.28335900 \\ \text { H } & 0.65427900 & 0.61517900 & 1.38638800 \\ \text { H } & 1.24681100 & 1.50952900 & -0.01689900 \\ \text { N } & 1.45942200 & -0.56411600 & -0.24036600 \\ \text { H } & 0.89151300 & -1.39002700 & -0.07046000 \\ \text { H } & 2.33699100 & -0.68962100 & 0.25308900\end{array}$

Stationary Point

\begin{tabular}{rlllll}
\hline $\mathbf{1 1}$ & $\mathrm{N}$ & -0.62432100 & 0.44152500 & 0.00000500 \\
& $\mathrm{H}$ & -0.23819600 & -0.49800400 & -0.00000200 \\
& $\mathrm{H}$ & -0.23817800 & 0.91128800 & 0.81365300 \\
& $\mathrm{H}$ & -0.23820700 & 0.91128800 & -0.81365600 \\
& $\mathbf{1 8 , 3 6}$ & $\mathrm{H}$ & 2.53787000 & -0.53254400 & 0.00000000 \\
& & & & \\
$\mathbf{1 9 , 2 8}$ & $\mathrm{N}$ & -1.82120100 & 0.53353200 & -0.05669500 \\
& $\mathrm{H}$ & -1.44720600 & -0.42547500 & 0.00037600 \\
& $\mathrm{H}$ & -1.44718900 & 0.96360400 & 0.80236100 \\
& & & & \\
& $\mathbf{2 0}$ & $\mathrm{N}$ & -2.85008700 & 3.15200300 & -0.05304800 \\
& $\mathrm{H}$ & -3.25382500 & 3.66908200 & 0.71640100 \\
& $\mathrm{H}$ & -2.30138600 & 3.75904800 & -0.64705900
\end{tabular}




$\begin{array}{ccccc} & \mathrm{C} & -2.22752800 & 1.95251200 & 0.30268900 \\ \mathrm{H} & -2.74029900 & 1.34620500 & 1.03892800 \\ \mathrm{H} & \mathrm{C} & -1.67828000 & 1.44651400 & -0.48145600 \\ & & & & \\ \mathrm{H} & -1.53727800 & 1.21845600 & 0.19787500 \\ \mathrm{H} & -2.55118300 & 1.45320100 & -0.14965000 \\ \mathrm{~N} & -0.87680100 & 0.18455500 & -0.11468200 \\ \mathrm{H} & -1.42048900 & -0.41280900 & -0.74347900 \\ & & & \\ \mathbf{3 7} & & & & \\ \mathrm{C} & 0.62793900 & -0.01192300 & 0.00000000 \\ \mathrm{H} & 1.12680800 & 0.95943700 & -0.00000100 \\ \mathrm{H} & 0.97219500 & -0.58171000 & -0.87883500 \\ \mathrm{~N} & -0.80505200 & 0.15291500 & 0.00000000 \\ \mathrm{H} & -1.20346700 & -0.79488900 & 0.00000000 \\ \mathrm{H} & 0.97219500 & -0.58170800 & 0.87883600\end{array}$


Table S2. Geometries for cationic species corresponding to stationary points calculated for this work. All calculations were conducted at the CBS-QB3 level.

\begin{tabular}{|c|c|c|c|c|}
\hline \multicolumn{5}{|l|}{ Stationary Point } \\
\hline \multirow[t]{12}{*}{1} & $\mathrm{~N}$ & 1.14904400 & -0.65461800 & -0.13466200 \\
\hline & $\mathrm{H}$ & 1.84627700 & -1.12086900 & 0.43736800 \\
\hline & $\mathrm{H}$ & 1.20917200 & -0.89528700 & -1.11871900 \\
\hline & $\mathrm{C}$ & 0.73160800 & 0.71505800 & 0.21019900 \\
\hline & $\mathrm{H}$ & 0.83417400 & 0.83191200 & 1.29026900 \\
\hline & $\mathrm{H}$ & 1.34946900 & 1.47613000 & -0.27472600 \\
\hline & $\mathrm{C}$ & -0.73159800 & 0.71507200 & -0.21015800 \\
\hline & $\mathrm{H}$ & -0.83403200 & 0.83194500 & -1.29024200 \\
\hline & $\mathrm{H}$ & -1.34950800 & 1.47614800 & 0.27469500 \\
\hline & $\mathrm{N}$ & -1.14903800 & -0.65463800 & 0.13460900 \\
\hline & $\mathrm{H}$ & -1.20900500 & -0.89534500 & 1.11867100 \\
\hline & $\mathrm{H}$ & -1.84665000 & -1.12061700 & -0.43719500 \\
\hline \multirow[t]{12}{*}{2} & $\mathrm{~N}$ & -1.23036000 & -0.71346100 & -0.08509500 \\
\hline & $\mathrm{H}$ & -1.68909400 & -0.96558800 & -0.95339400 \\
\hline & $\mathrm{H}$ & -1.87877500 & -0.89713000 & 0.67235900 \\
\hline & $\mathrm{C}$ & -0.74301000 & 0.67003800 & -0.08660400 \\
\hline & $\mathrm{H}$ & -0.98651600 & 1.17663200 & -1.02054100 \\
\hline & $\mathrm{H}$ & -1.19003100 & 1.25015100 & 0.72069700 \\
\hline & $\mathrm{C}$ & 0.81404000 & 0.67342500 & 0.09520900 \\
\hline & $\mathrm{H}$ & 1.15260800 & 1.23862400 & 0.98511700 \\
\hline & $\mathrm{H}$ & 1.35457300 & 1.17516500 & -0.73037300 \\
\hline & $\mathrm{N}$ & 1.27458300 & -0.65203000 & 0.19914800 \\
\hline & $\mathrm{H}$ & 0.46266000 & -1.33166600 & 0.13322000 \\
\hline & $\mathrm{H}$ & 2.25395500 & -0.90496600 & 0.31986600 \\
\hline \multirow[t]{2}{*}{3} & $\mathrm{~N}$ & 1.64159000 & -0.48367700 & 0.10521800 \\
\hline & $\mathrm{H}$ & 1.68956300 & -1.37431000 & -0.36855600 \\
\hline
\end{tabular}




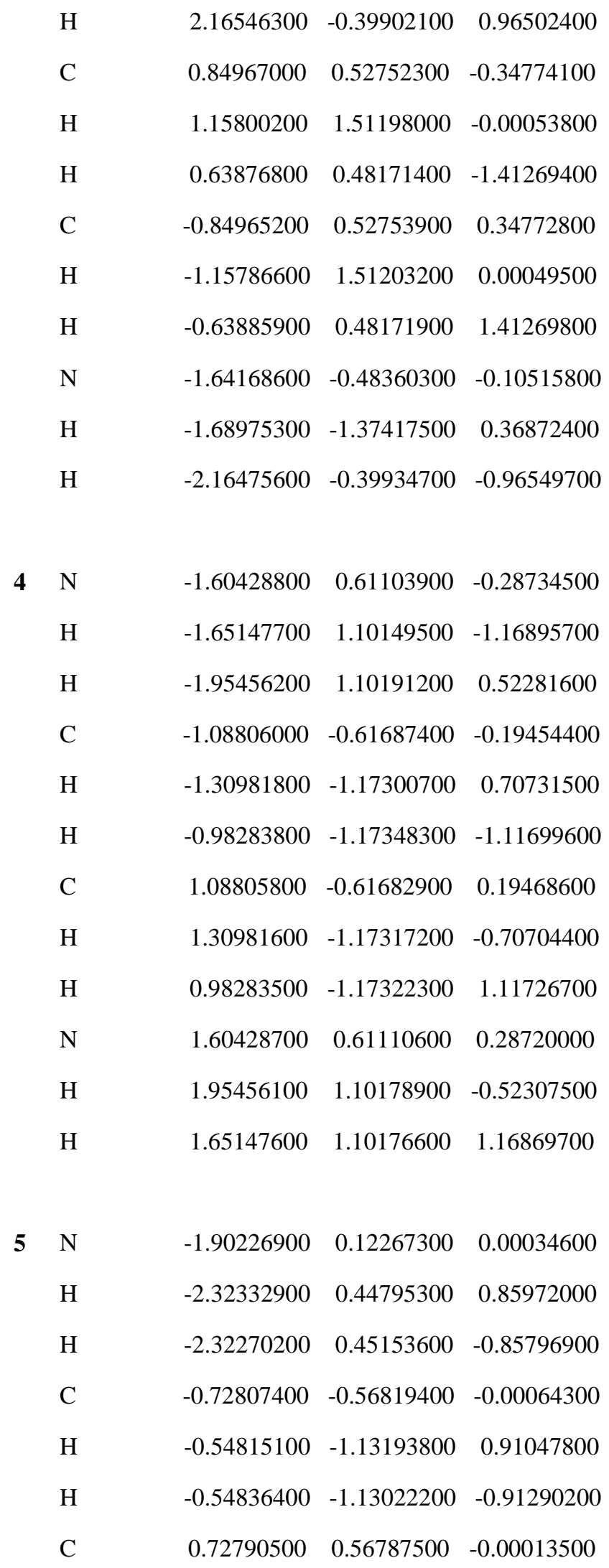




$\begin{array}{llll}\mathrm{H} & 0.54763400 & 1.13067900 & 0.91150800 \\ \mathrm{H} & 0.54816800 & 1.13081300 & -0.91182400 \\ \mathrm{~N} & 1.90261800 & -0.12214500 & 0.00031400 \\ \mathrm{H} & 2.32238800 & -0.45013000 & 0.85931400 \\ \mathrm{H} & 2.32292300 & -0.45048000 & -0.85827700\end{array}$

$\begin{array}{lllll}6 & \mathrm{~N} & 1.83021400 & -0.07425200 & -0.15677500\end{array}$

$\mathrm{H} \quad \begin{array}{llll}1.89852100 & 0.15997400 & -1.13860900\end{array}$

$\begin{array}{llll}\mathrm{H} & 2.64374300 & -0.51116700 & 0.25673000\end{array}$

$\begin{array}{llll}\text { C } & 0.63850900 & -0.07551000 & 0.49798400\end{array}$

$\mathrm{H} \quad \begin{array}{llll}0.21982700 & -1.13498000 & 0.30549100\end{array}$

$\mathrm{H} \quad \begin{array}{llll}0.72564500 & -0.09922900 & 1.58517100\end{array}$

$\begin{array}{llll}\text { C } & -0.57034600 & 0.65843700 & -0.07535800\end{array}$

$\begin{array}{llll}\mathrm{H} & -0.40532100 & 1.03298800 & -1.08580700\end{array}$

$\begin{array}{llll}\mathrm{H} & -0.94424200 & 1.47128700 & 0.54509400\end{array}$

N $\quad-1.49976300 \quad-0.51823000 \quad-0.09796000$

$\begin{array}{llll}\mathrm{H} & -2.23168500 & -0.51822000 & 0.60833200\end{array}$

$\begin{array}{llll}\mathrm{H} & -1.87735400 & -0.77268200 & -1.00724500\end{array}$

$\begin{array}{lllll}7 & \mathrm{~N} & -1.76755300 & -0.52548700 & 0.13341800\end{array}$

$\begin{array}{llll}\mathrm{H} & -1.71921100 & -0.81011500 & 1.10352900\end{array}$

$\mathrm{H} \quad-2.54966700 \quad-0.87211900 \quad-0.40505300$

$\begin{array}{llll}\text { C } & -0.90351800 & 0.34113300 & -0.38932300\end{array}$

$\mathrm{H} \quad-1.07637400 \quad 0.60725600 \quad-1.42630300$

$\begin{array}{llll}\text { C } & 0.18219500 & 0.87634200 & 0.29984300\end{array}$

$\begin{array}{llll}\mathrm{H} & 0.28371400 & 0.72999700 & 1.36746800\end{array}$

$\begin{array}{llll}\mathrm{H} & 0.71477700 & 1.70669900 & -0.13813000\end{array}$

$\begin{array}{llll}\mathrm{N} & 2.01877500 & -0.44419000 & -0.07397600\end{array}$

$\begin{array}{llll}\mathrm{H} & 2.25122300 & -0.43983900 & -1.06319600\end{array}$

$\begin{array}{llll}\mathrm{H} & 1.87605400 & -1.40241800 & 0.23229900\end{array}$

$\begin{array}{lllll}\mathrm{H} & 2.78887100 & -0.03657000 & 0.45017000\end{array}$ 


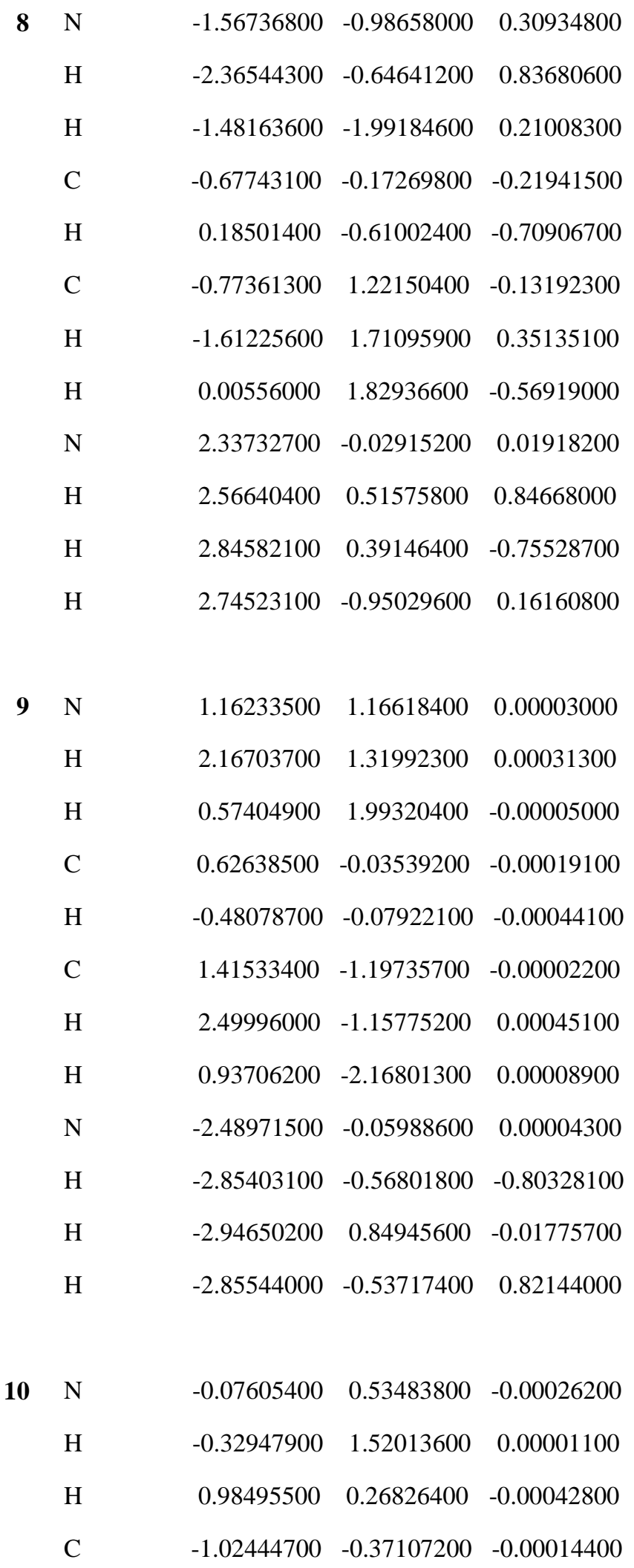




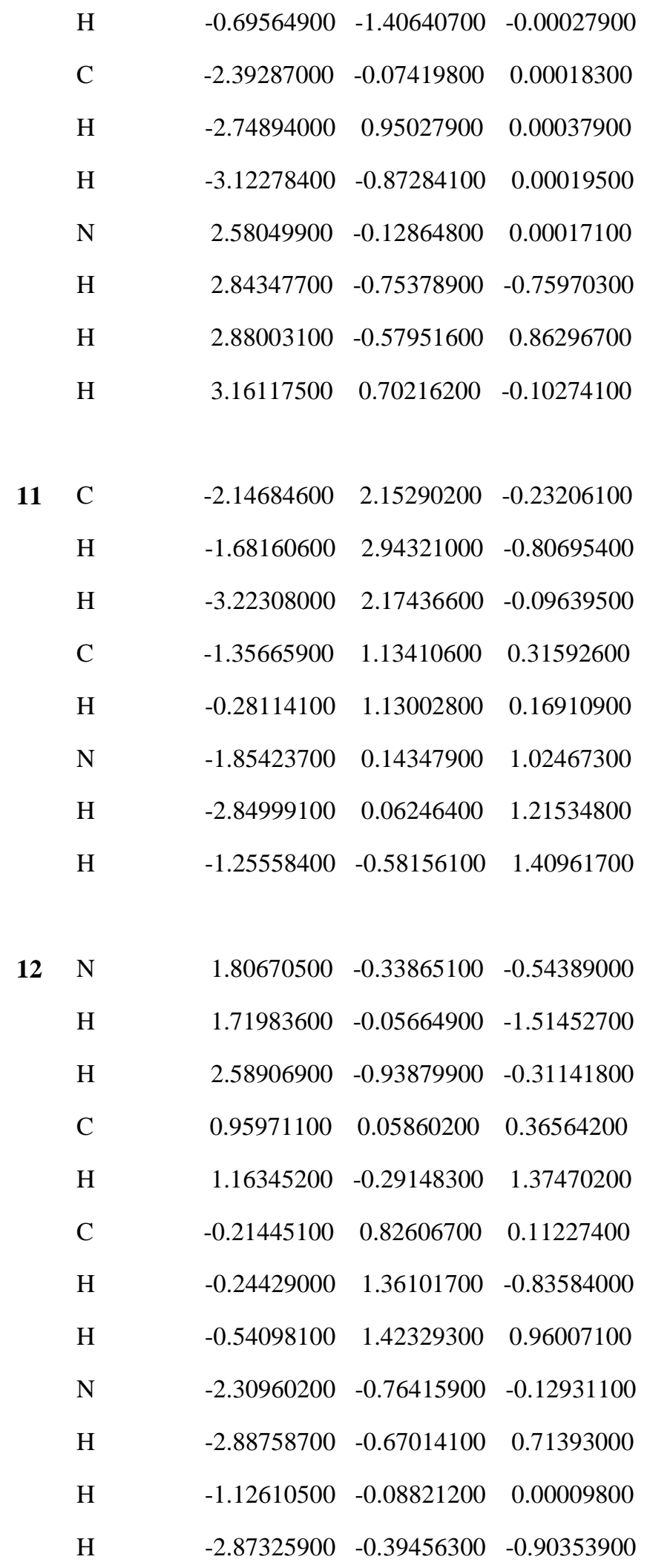




\begin{tabular}{|c|c|c|c|c|}
\hline \multirow[t]{4}{*}{13} & $\mathrm{~N}$ & 2.01987600 & -0.54358500 & -0.19906500 \\
\hline & $\mathrm{H}$ & 2.17509800 & -0.36128400 & -1.18711300 \\
\hline & $\mathrm{H}$ & 2.65081400 & -1.20097700 & 0.24880100 \\
\hline & $\mathrm{C}$ & 1.06473800 & 0.03311200 & 0.45064500 \\
\hline & $\mathrm{H}$ & 0.98635400 & -0.22508700 & 1.50498300 \\
\hline & $\mathrm{C}$ & 0.09532800 & 0.94656200 & -0.13514300 \\
\hline & $\mathrm{H}$ & 0.32710000 & 1.23440500 & -1.16083400 \\
\hline & $\mathrm{H}$ & -0.01296000 & 1.82979400 & 0.50301100 \\
\hline & $\mathrm{N}$ & -2.78941200 & -0.38481000 & -0.03013000 \\
\hline & $\mathrm{H}$ & -3.27101100 & -0.94212200 & -0.74684600 \\
\hline & $\mathrm{H}$ & -3.52783700 & -0.15477200 & 0.64677000 \\
\hline & $\mathrm{H}$ & -0.90120300 & 0.44076600 & -0.09741500 \\
\hline \multirow[t]{12}{*}{14} & $\mathrm{C}$ & -0.85118700 & -0.34728800 & 0.00133500 \\
\hline & $\mathrm{H}$ & -0.49983500 & -1.37808800 & 0.00490700 \\
\hline & $\mathrm{N}$ & 2.77159900 & -0.14401700 & 0.00017700 \\
\hline & $\mathrm{H}$ & 3.37983900 & -0.25965600 & 0.81828900 \\
\hline & $\mathrm{H}$ & 3.37260900 & -0.28276200 & -0.81969100 \\
\hline & $\mathrm{C}$ & -2.30180800 & -0.08832800 & -0.00010800 \\
\hline & $\mathrm{H}$ & -2.75069800 & -0.57302900 & 0.87464100 \\
\hline & $\mathrm{H}$ & -2.54960200 & 0.97321400 & -0.00258500 \\
\hline & $\mathrm{H}$ & -2.74914600 & -0.57661700 & -0.87369600 \\
\hline & $\mathrm{N}$ & 0.05970400 & 0.55353100 & -0.00095100 \\
\hline & $\mathrm{H}$ & -0.19659300 & 1.53828000 & -0.00394800 \\
\hline & $\mathrm{H}$ & 1.09227400 & 0.30575100 & 0.00013700 \\
\hline \multirow[t]{5}{*}{15} & $\mathrm{C}$ & -0.54821400 & -0.07289500 & -0.41522000 \\
\hline & $\mathrm{H}$ & -0.02183200 & -0.16902200 & -1.35645300 \\
\hline & $\mathrm{N}$ & 2.11314800 & -0.05110900 & 0.06966700 \\
\hline & $\mathrm{H}$ & 2.88700800 & 0.19422700 & -0.56148800 \\
\hline & $\mathrm{H}$ & 2.58020300 & -0.29572300 & 0.95231600 \\
\hline
\end{tabular}




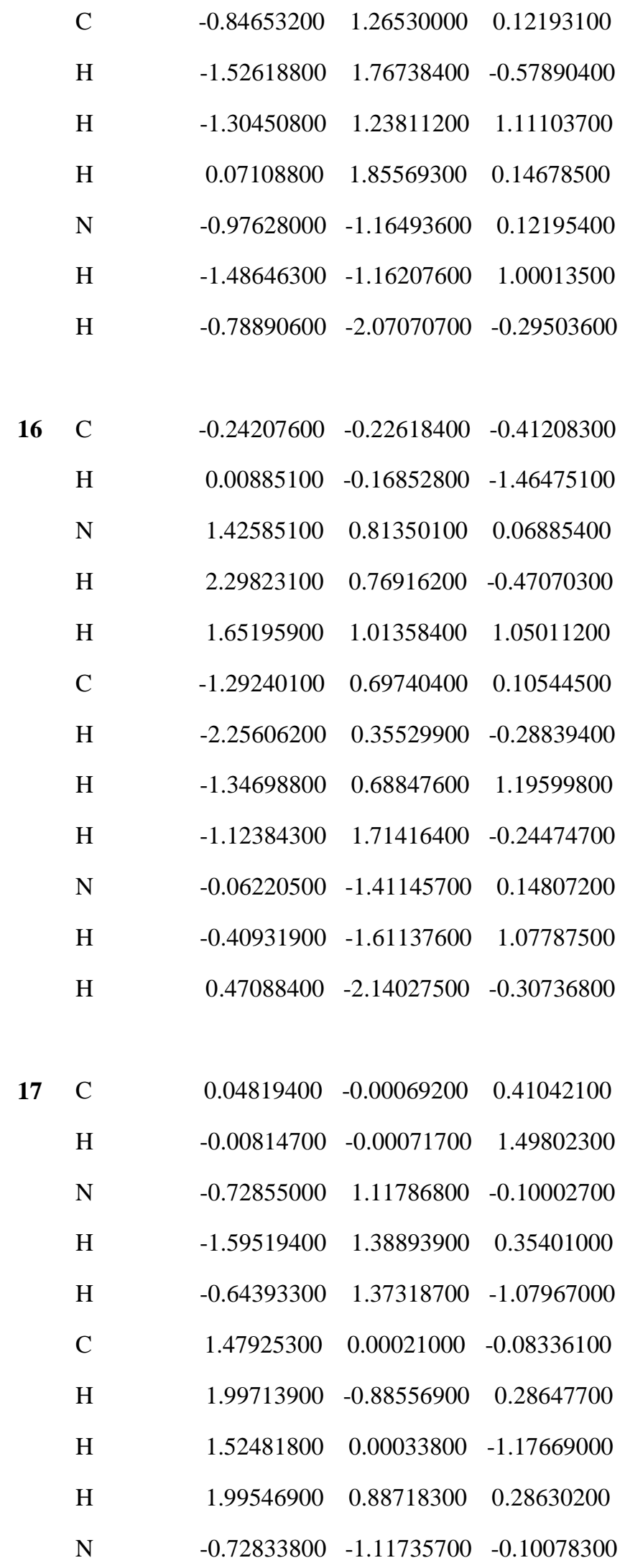




\begin{tabular}{|c|c|c|c|c|}
\hline & $\mathrm{H}$ & -0.64380200 & -1.37237000 & -1.08037600 \\
\hline & $\mathrm{H}$ & -1.59281000 & -1.39167000 & 0.35523700 \\
\hline 18 & $\mathrm{C}$ & -0.05838900 & 0.00012100 & -0.01098500 \\
\hline & $\mathrm{N}$ & -0.70476300 & -1.15099900 & -0.00161300 \\
\hline & $\mathrm{H}$ & -1.71416000 & -1.21457200 & 0.02446400 \\
\hline & $\mathrm{H}$ & -0.19406200 & -2.02203700 & -0.01143300 \\
\hline & $\mathrm{C}$ & 1.43735100 & 0.00239700 & 0.00176800 \\
\hline & $\mathrm{H}$ & 1.83224400 & 0.89665300 & -0.47982900 \\
\hline & $\mathrm{H}$ & 1.78412700 & -0.01436600 & 1.03977600 \\
\hline & $\mathrm{H}$ & 1.83393800 & -0.87550400 & -0.50814000 \\
\hline & $\mathrm{N}$ & -0.70892300 & 1.14880400 & -0.00165300 \\
\hline & $\mathrm{H}$ & -1.71857600 & 1.20838000 & 0.02430900 \\
\hline & $\mathrm{H}$ & -0.20147600 & 2.02170000 & -0.01098100 \\
\hline 19 & $\mathrm{~N}$ & -2.94837800 & 1.38083000 & -0.14826100 \\
\hline & $\mathrm{H}$ & -2.52138500 & 2.23040700 & 0.21539000 \\
\hline & $\mathrm{H}$ & -3.94928800 & 1.41434900 & -0.32489900 \\
\hline & $\mathrm{C}$ & -2.25698700 & 0.31933700 & -0.37277900 \\
\hline & $\mathrm{H}$ & -2.80500800 & -0.53717100 & -0.76146300 \\
\hline & $\mathrm{C}$ & -0.81233300 & 0.20662800 & -0.13931100 \\
\hline & $\mathrm{H}$ & -0.63319700 & -0.62766300 & 0.55075300 \\
\hline & $\mathrm{H}$ & -0.32720100 & -0.08344600 & -1.07996200 \\
\hline & $\mathrm{H}$ & -0.35973000 & 1.11841200 & 0.24990900 \\
\hline 20 & $\mathrm{~N}$ & -2.68021900 & 3.16314000 & 0.06552200 \\
\hline & $\mathrm{H}$ & -3.31716500 & 3.63221800 & 0.70959700 \\
\hline & $\mathrm{H}$ & -2.33284900 & 3.72130500 & -0.71444900 \\
\hline & $\mathrm{C}$ & -2.33705100 & 1.94579100 & 0.22656700 \\
\hline & $\mathrm{H}$ & -2.72751600 & 1.38299100 & 1.06926600 \\
\hline & $\mathrm{H}$ & -1.65660500 & 1.47991700 & -0.48004700 \\
\hline
\end{tabular}


21 N

$\mathrm{H}$

$\mathrm{H}$

C

$\mathrm{H}$

H

C

$\mathrm{H}$

H

$\mathrm{N}$

$\mathrm{H}$

$\mathrm{H}$

22 N

$\mathrm{H}$

$\mathrm{H}$

C

$\mathrm{H}$

$\mathrm{H}$

C

$\mathrm{H}$

$\mathrm{H}$

$\mathrm{N}$

$\mathrm{H}$

$\mathrm{H}$

23 C

H

$\mathrm{N}$

$\mathrm{H}$ $\begin{array}{lll}1.41994800 & -0.62138900 & 0.01884500\end{array}$

$\begin{array}{lll}2.03208400 & -1.28731900 & 0.48210600\end{array}$

$\begin{array}{lll}0.44208700 & -0.91016200 & -0.19928200\end{array}$

$\begin{array}{llll}1.74436200 & 0.61784600 & -0.08349400\end{array}$

$\begin{array}{lll}2.69113000 & 0.97193600 & 0.31028900\end{array}$

$\begin{array}{llll}1.05765000 & 1.30143400 & -0.56691200\end{array}$

$\begin{array}{lll}-1.57670000 & 0.75268100 & 0.12581500\end{array}$

$\begin{array}{llll}-1.75046000 & 1.39419400 & -0.72504200\end{array}$

$\begin{array}{lll}-1.64155600 & 1.12564100 & 1.13635200\end{array}$

$\begin{array}{lll}-1.45274100 & -0.61673000 & -0.07976100\end{array}$

$\begin{array}{lll}-1.83818400 & -0.95526700 & -0.95655400\end{array}$

$\begin{array}{lll}-1.76917600 & -1.19679300 & 0.69152900\end{array}$

$\begin{array}{lll}1.36678200 & -0.56062000 & 0.00008100\end{array}$

$\begin{array}{lll}1.95622600 & -1.39141300 & -0.00076200\end{array}$

$\begin{array}{lll}0.28495300 & -0.68479400 & 0.00064600\end{array}$

$\begin{array}{llll}1.89791900 & 0.59503000 & -0.00000800\end{array}$

$\begin{array}{llll}2.97780800 & 0.71560300 & -0.00094600\end{array}$

$\begin{array}{lll}1.24953600 & 1.46653800 & 0.00081500\end{array}$

$\begin{array}{llll}-1.72799900 & 0.74577300 & -0.00015900\end{array}$

$\begin{array}{llll}-1.94355300 & 1.22309200 & -0.94343500\end{array}$

$\begin{array}{lll}-1.94143200 & 1.22373300 & 0.94328300\end{array}$

$\begin{array}{lll}-1.38730700 & -0.63257800 & 0.00010000\end{array}$

$\begin{array}{lll}-1.72949900 & -1.12265600 & -0.82380700\end{array}$

$\begin{array}{lll}-1.72988300 & -1.12253500 & 0.82394200\end{array}$

$\begin{array}{lll}0.01086500 & -0.82670300 & 0.00000000\end{array}$

$\begin{array}{lll}-0.08714600 & -1.30953800 & 0.95960600\end{array}$

$\begin{array}{lll}0.01086500 & 0.64286800 & 0.00000000\end{array}$

$\begin{array}{llll}0.48832500 & 1.02092300 & -0.82774800\end{array}$ 


$\begin{array}{llll}\mathrm{H} & -0.08714600 & -1.30953800 & -0.95960600 \\ \mathrm{H} & -0.94359800 & 1.03737500 & 0.00000000 \\ \mathrm{H} & 0.48832500 & 1.02092300 & 0.82774800\end{array}$

$24 \mathrm{~N} \quad 2.18080800 \quad 0.00772400-0.00140400$

$\begin{array}{llll}\mathrm{H} & 2.76848000 & 0.13920000 & -0.83225400\end{array}$

$\begin{array}{llll}\mathrm{H} & 2.75406100 & 0.18853700 & 0.83023800\end{array}$

$\begin{array}{lllll}\text { C } & 0.13694000 & -0.50486200 & -0.00327200\end{array}$

$\mathrm{H} \quad 0.26286700 \quad-1.04013400-0.93186000$

$\begin{array}{llll}\mathrm{H} & 0.26463800 & -1.05170700 & 0.91835600\end{array}$

$\begin{array}{llll}\text { C } & -0.65794200 & 0.73647400 & 0.00520700\end{array}$

$\begin{array}{llll}\mathrm{H} & -0.61723500 & 1.35159300 & -0.88920000\end{array}$

$\mathrm{H} \quad-0.61656500 \quad 1.33956400 \quad 0.90775000$

$\begin{array}{llll}\mathrm{N} & -1.74413600 & -0.24333300 & -0.00065600\end{array}$

$\mathrm{H} \quad-2.27590600 \quad-0.36866700 \quad 0.85006500$

$\begin{array}{llll}\mathrm{H} & -2.27574600 & -0.35823400 & -0.85294600\end{array}$

$\begin{array}{lllll}25 & \mathrm{~N} & -2.59057400 & -0.02376900 & 0.00009300\end{array}$

$\begin{array}{llll}\mathrm{H} & -3.22587900 & 0.05819000 & 0.80502200\end{array}$

$\begin{array}{llll}\mathrm{H} & -3.22605700 & 0.05783900 & -0.80473100\end{array}$

$\begin{array}{llll}\text { C } & 0.25130700 & -0.49714800 & 0.00000100\end{array}$

$\begin{array}{llll}\mathrm{H} & -0.13272900 & -0.91976100 & 0.91617000\end{array}$

$\mathrm{H} \quad-0.13273200 \quad-0.91989200 \quad-0.91610700$

$\begin{array}{lllll}\text { C } & 0.80713300 & 0.86101900 & -0.00010100\end{array}$

$\begin{array}{llll}\mathrm{H} & 0.82321900 & 1.43398100 & 0.91767800\end{array}$

$\begin{array}{llll}\mathrm{H} & 0.82321200 & 1.43384300 & -0.91796500\end{array}$

$\begin{array}{llll}\mathrm{N} & 1.75788100 & -0.30639800 & -0.00001700\end{array}$

$\begin{array}{llll}\mathrm{H} & 2.27458800 & -0.50819200 & -0.85123900\end{array}$

$\begin{array}{llll}\mathrm{H} & 2.27458800 & -0.50806800 & 0.85123500\end{array}$

$\begin{array}{lllll}26 & \mathrm{~N} & 2.36058200 & 0.83576300 & -0.97173400\end{array}$ 


$\begin{array}{lrrr}\mathrm{H} & 3.24088400 & 0.67747200 & -0.46396200 \\ \mathrm{H} & 2.65687000 & 1.39592200 & -1.78208700 \\ \mathrm{C} & -0.50937700 & -0.42256500 & 1.12606500 \\ \mathrm{H} & -0.01137300 & -1.35242900 & 1.36770500 \\ \mathrm{H} & -0.70599900 & 0.24182600 & 1.95741800 \\ \mathrm{C} & -0.41732100 & 0.11749300 & -0.23884600 \\ \mathrm{H} & 0.15884000 & -0.40831100 & -0.98461600 \\ \mathrm{H} & -0.54852100 & 1.17731500 & -0.40533800 \\ \mathrm{~N} & -1.68209200 & -0.58927000 & 0.19918800 \\ \mathrm{H} & -2.51648100 & -0.03575100 & 0.37229200 \\ \mathrm{H} & -1.87337300 & -1.51412600 & -0.17564600\end{array}$

$\begin{array}{lllll}27 & \mathrm{~N} & -2.39445300 & -0.00003500 & -0.10084100\end{array}$

$\begin{array}{llll}\mathrm{H} & -3.00080800 & 0.81858200 & -0.22321400\end{array}$

$\mathrm{H} \quad-3.00006800 \quad-0.81893500 \quad-0.22496900$

$\begin{array}{llll}\text { C } & 1.25962200 & -0.73854200 & -0.28062600\end{array}$

$\mathrm{H} \quad \begin{array}{llll}0.77171500 & -1.27129600 & -1.08621700\end{array}$

$\begin{array}{llll}\mathrm{H} & 2.04535700 & -1.27184200 & 0.23781500\end{array}$

$\begin{array}{lllll}\text { C } & 1.25980900 & 0.73817500 & -0.28135500\end{array}$

$\begin{array}{llll}\mathrm{H} & 0.77206400 & 1.27027600 & -1.08747400\end{array}$

$\begin{array}{llll}\mathrm{H} & 2.04568500 & 1.27175500 & 0.23658700\end{array}$

$\begin{array}{llll}\mathrm{N} & 0.31537200 & 0.00038000 & 0.60909600\end{array}$

$\begin{array}{llll}\mathrm{H} & 0.51772000 & 0.00086000 & 1.60448000\end{array}$

$\begin{array}{llll}\mathrm{H} & -0.71468000 & 0.00038500 & 0.35709300\end{array}$

$\begin{array}{lllll}28 & \mathrm{~N} & -2.41224000 & 1.26502100 & -0.05162100\end{array}$

$\begin{array}{llll}\mathrm{H} & -2.56803900 & 1.77457400 & 0.81483200\end{array}$

$\begin{array}{llll}\mathrm{H} & -2.75776700 & 1.74720500 & -0.87791700\end{array}$

$\begin{array}{llll}\text { C } & -2.47525500 & -0.23612300 & -0.02029400\end{array}$

$\mathrm{H} \quad \begin{array}{llll}\mathrm{H} & -2.75859400 & -0.63976100 & 0.94312500\end{array}$

$\begin{array}{llll}\mathrm{H} & -2.96335900 & -0.66927600 & -0.88372500\end{array}$ 


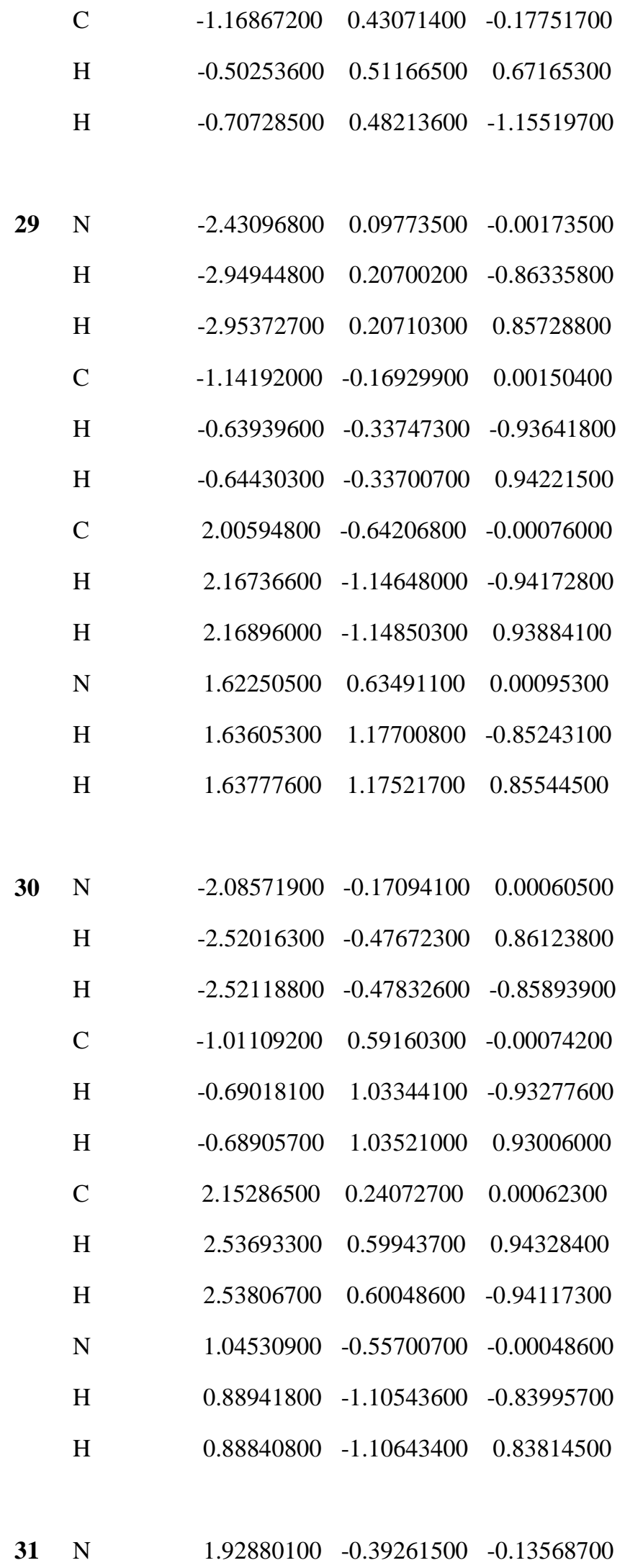




$\begin{array}{llll}\mathrm{H} & 2.52110500 & -0.05844900 & -0.88433300 \\ \mathrm{H} & 1.99831000 & -1.37548100 & 0.09264300 \\ \mathrm{C} & 1.10986600 & 0.40951900 & 0.51234500 \\ \mathrm{H} & 0.62799000 & 0.04535700 & 1.40755000 \\ \mathrm{H} & 1.19063300 & 1.47412100 & 0.34032200 \\ \mathrm{C} & -2.01148700 & -0.44937900 & 0.12648900 \\ \mathrm{H} & -2.58725800 & -0.13021300 & 0.98177600 \\ \mathrm{H} & -2.03988200 & -1.46936800 & -0.22524100 \\ \mathrm{~N} & -1.10273400 & 0.41066200 & -0.41429600 \\ \mathrm{H} & -0.80067000 & 0.20664400 & -1.36143300 \\ \mathrm{H} & -1.29284800 & 1.39883000 & -0.28144800\end{array}$

$32 \mathrm{~N}$

$\begin{array}{lll}1.38346800 & 0.63255900 & -0.00009200\end{array}$

$\begin{array}{llll}\mathrm{H} & 1.50171500 & 1.15741700 & 0.85568500\end{array}$

$\mathrm{H} \quad \begin{array}{llll}1.49962800 & 1.15724100 & -0.85625400\end{array}$

$\begin{array}{llll}\text { C } & 1.43073200 & -0.69163700 & 0.00000400\end{array}$

$\mathrm{H} \quad 1.49726600 \quad-1.22028700 \quad-0.93910300$

$\begin{array}{llll}\mathrm{H} & 1.50029900 & -1.22008600 & 0.93901400\end{array}$

$\begin{array}{llll}\text { C } & -1.43126400 & 0.69162500 & 0.00013300\end{array}$

$\mathrm{H} \quad-1.49954600 \quad 1.22011800 \quad-0.93893500$

$\begin{array}{llll}\mathrm{H} & -1.49997900 & 1.21994200 & 0.93927100\end{array}$

$\begin{array}{llll}\mathrm{N} & -1.38307900 & -0.63248600 & 0.00003500\end{array}$

$\begin{array}{lllll}\mathrm{H} & -1.49977000 & -1.15746300 & 0.85594100\end{array}$

$\begin{array}{lllll}\mathrm{H} & -1.49913700 & -1.15732000 & -0.85604400\end{array}$

$33 \mathrm{~N}$

$\begin{array}{lll}0.74622500 & -0.57723100 & 0.09815400\end{array}$

$\begin{array}{llll}\mathrm{H} & 1.27290100 & -1.44569800 & 0.00022900\end{array}$

$\mathrm{H} \quad \begin{array}{llll}\mathrm{H} & 1.14888400 & 0.05807100 & 1.09481700\end{array}$

$\begin{array}{lllll}\text { C } & 1.51059900 & 0.64488100 & -0.03948800\end{array}$

$\begin{array}{llll}\mathrm{H} & 0.92297600 & 1.53721100 & -0.19587600\end{array}$

$\begin{array}{lllll}\mathrm{H} & 2.55903100 & 0.54736200 & -0.28400900\end{array}$ 


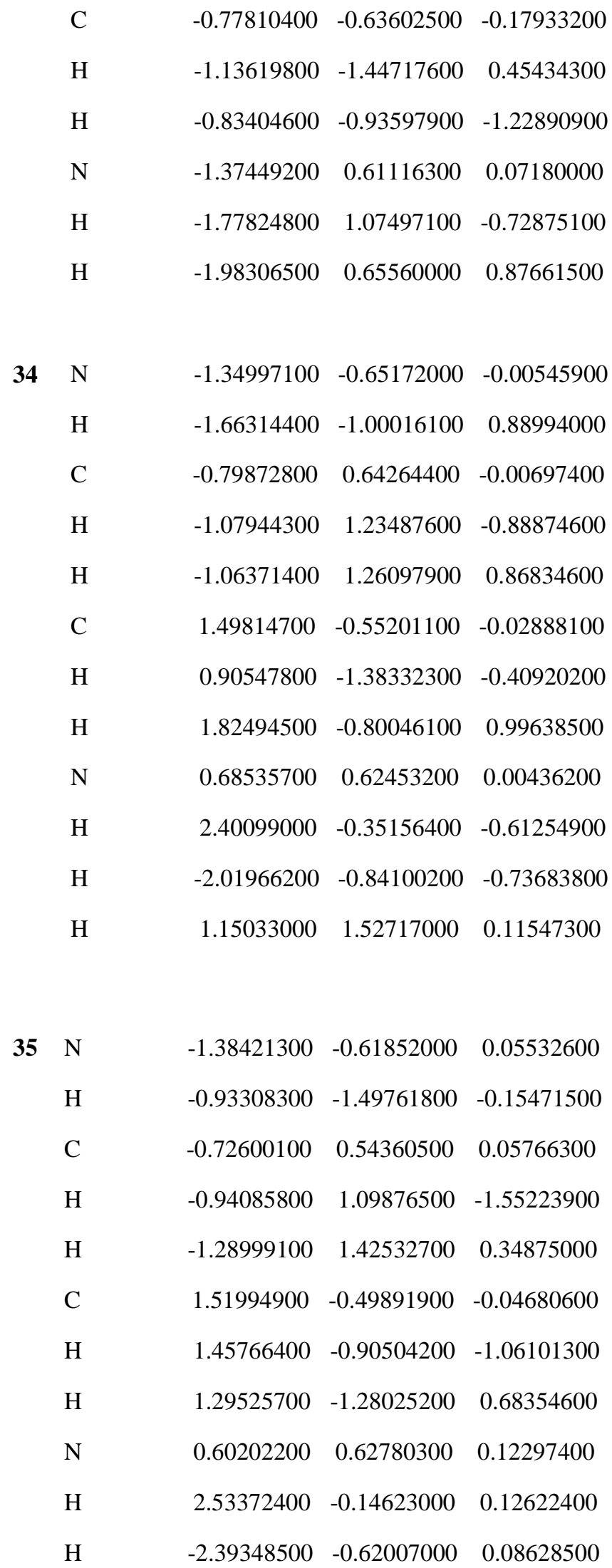


$\begin{array}{llll}\mathrm{H} & 0.99357200 & 1.55788000 & 0.19035500\end{array}$

\begin{tabular}{rrrr}
36 & -1.42537600 & -0.54131900 & -0.00001200 \\
$\mathrm{H}$ & -2.43508400 & -0.51025100 & -0.00000400 \\
$\mathrm{C}$ & -0.73252900 & 0.57853400 & 0.00000100 \\
$\mathrm{H}$ & -1.28935300 & 1.50930900 & 0.00007000 \\
$\mathrm{H}$ & 1.33477100 & -1.10659700 & -0.89729900 \\
$\mathrm{C}$ & 1.48594900 & -0.50227100 & -0.00000600 \\
$\mathrm{H}$ & 2.50771000 & -0.13144600 & 0.00105800 \\
$\mathrm{H}$ & 1.33342100 & -1.10771500 & 0.89629500 \\
$\mathrm{~N}$ & 0.57429600 & 0.65087900 & 0.00000800 \\
$\mathrm{H}$ & 0.97894700 & 1.57775200 & -0.00008700 \\
$\mathrm{H}$ & -0.99337100 & -1.45555700 & 0.00003100 \\
& & & \\
\hline $\mathrm{N}$ & -2.68021900 & 3.16314000 & 0.06552200 \\
$\mathrm{H}$ & -3.31716500 & 3.63221800 & 0.70959700 \\
$\mathrm{H}$ & -2.33284900 & 3.72130500 & -0.71444900 \\
$\mathrm{C}$ & -2.33705100 & 1.94579100 & 0.22656700 \\
$\mathrm{H}$ & -2.72751600 & 1.38299100 & 1.06926600 \\
$\mathrm{H}$ & -1.65660500 & 1.47991700 & -0.48004700
\end{tabular}




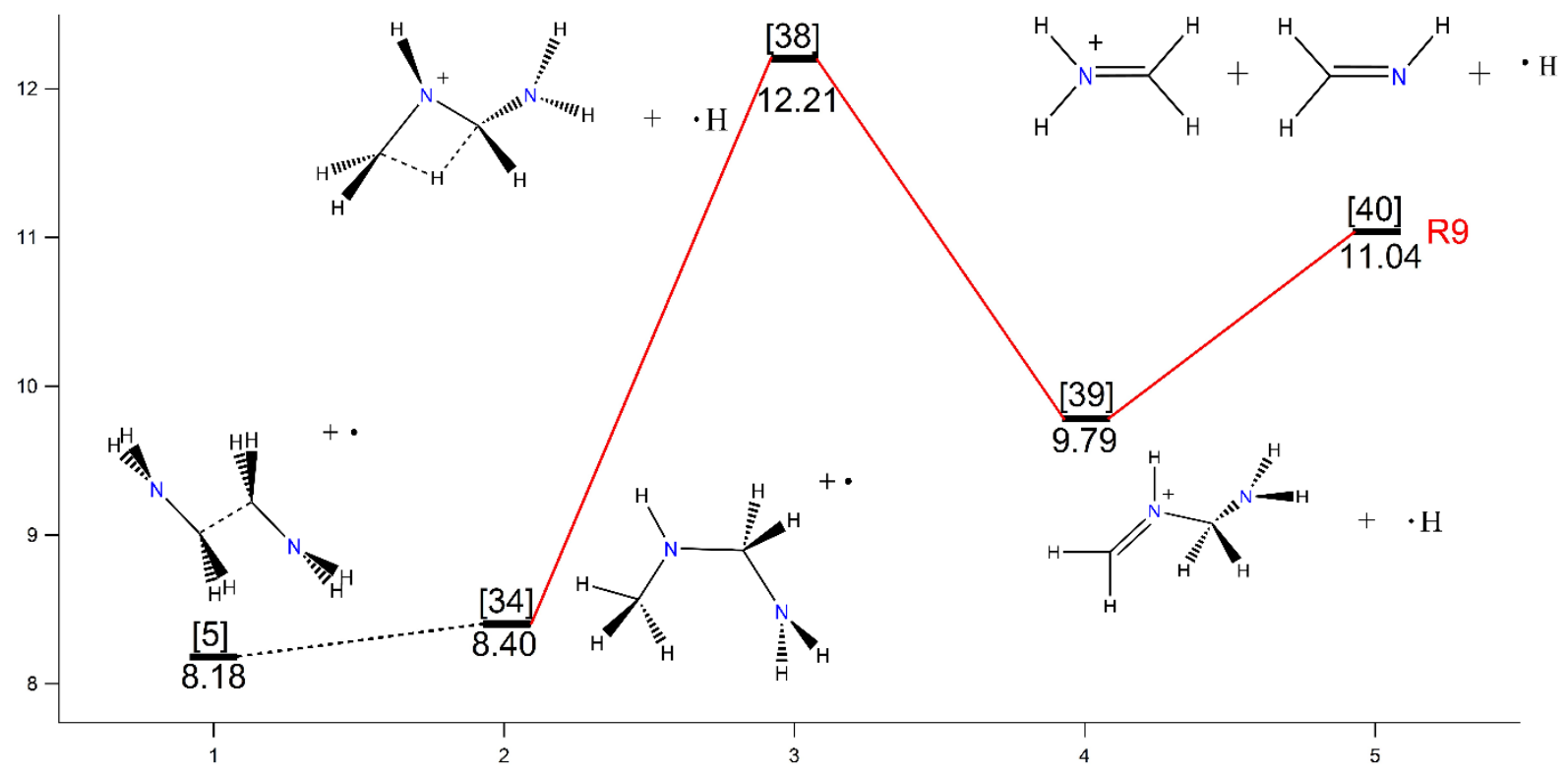

Figure S6. CBS-QB3 calculated potential energy surface diagram of possible dissociation channel R9 corresponding to formation of third $\mathrm{m} / \mathrm{z}=30$ appearance from ethylenediamine photodissociation. For energies, refer to Figure S7. 
[38]

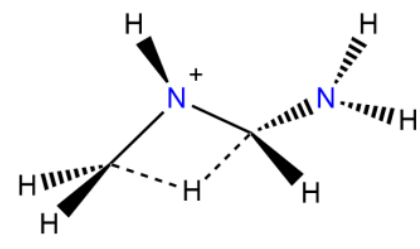

$-189.216080 /-0.49982$
[39]

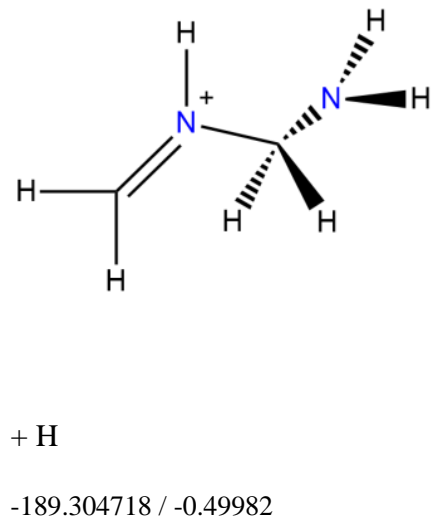

[40]<smiles>C=[NH+]</smiles><smiles>C=N</smiles>

$+\mathrm{H}$

Figure S7. CBS-QB3 calculated structures and energies at $0 \mathrm{~K}$ of stationary points [38] - [40] involved in the third $m / z=30(\mathbf{R 9})$ channel as described in Figure S6. Structures and energies of [5] and [34] are provided in Figure S5. 
Table S3. Ancillary Table of Thermochemical Values Used for Isodesmic Reaction Networks

\begin{tabular}{|c|c|}
\hline Species & $\Delta_{\mathrm{f}} H^{\ominus}{ }_{298} /\left(\mathrm{kJ} \mathrm{mol}^{-1}\right)^{1-3}$ \\
\hline $\mathrm{CH}_{3}$ & $146.427 \pm 0.080$ \\
\hline $\mathrm{CH}_{4}$ & $-74.533 \pm 0.057$ \\
\hline $\mathrm{CH}_{2} \mathrm{CH}_{2}$ & $52.53 \pm 0.14$ \\
\hline $\mathrm{CH}_{3} \mathrm{CH}_{3}$ & $-83.75 \pm 0.15$ \\
\hline $\mathrm{CH}_{2} \mathrm{CHCH}_{3}$ & $20.26 \pm 0.29$ \\
\hline $\mathrm{CH}_{3} \mathrm{CH}_{2} \mathrm{CH}_{2} \mathrm{CH}_{3}$ & $-125.82 \pm 0.34$ \\
\hline $\mathrm{CH}_{2} \mathrm{NH}_{2}$ & $148.7 \pm 1.1$ \\
\hline $\mathrm{CH}_{3} \mathrm{NHCH}_{3}$ & $-15.88 \pm 0.69$ \\
\hline $\mathrm{CH}_{3} \mathrm{NH}_{2}$ & $-20.91 \pm 0.53$ \\
\hline $\mathrm{CH}_{3} \mathrm{OH}$ & $-200.72 \pm 0.17$ \\
\hline $\mathrm{CH}_{2} \mathrm{CO}$ & $-48.56 \pm 0.15$ \\
\hline HNCO & $-119.03 \pm 0.37$ \\
\hline $\mathrm{HNO}$ & $106.93 \pm 0.11$ \\
\hline $\mathrm{H}_{2} \mathrm{NO}^{+}$ & $939.18 \pm 0.84$ \\
\hline $\mathrm{HOCH}_{2} \mathrm{CH}_{2} \mathrm{OH}$ & $-389.42 \pm 0.49$ \\
\hline $\mathrm{NH}_{3}$ & $-45.561 \pm 0.030$ \\
\hline $\mathrm{NH}_{4}{ }^{+}$ & $631.71 \pm 0.21$ \\
\hline
\end{tabular}




\section{Supplemental Computational Information}

The first rotational barrier [2] from the highest-energy cationic configuration [1] is 0.02 $\mathrm{eV}$ and leads to rotamer [3] at $0.37 \mathrm{eV}$ lower in energy than the original parent conformation. Rotational barrier [4] lies $0.14 \mathrm{eV}$ above [3] and is the transition to the anti-configuration of EN [5], $0.39 \mathrm{eV}$ lower in energy than the original parent ion.

The 1,3-hydrogen migration barrier [6] ${ }^{\ddagger}$ is calculated at $9.13 \mathrm{eV}$ and leads to a low-energy van der Waals product complex [7]. This intermediate leads to three distinct dissociation pathways R1-R3 $(m / z=43,59$, and 44), where the subsequent barriers are all less than the initial $9.13 \mathrm{eV}$ CBS-QB3 barrier. In addition, the relative energies of the products are also lower in energy than the original $9.13 \mathrm{eV}$ CBS-QB3 barrier $(8.41 \mathrm{eV}, 8.21 \mathrm{eV}$, and $8.98 \mathrm{eV}$, respectively). Thus, this transition is the highest-energy saddle point in these unimolecular reactions and has an influential role in the appearance energies of the fragments.

\section{Dissociation Channels}

R1) The functional group migration process [7-10] occurs in a low-energy realm relative to the other two processes ( $\mathbf{R} 2$ and $\mathbf{R 3}$ ) originating from [7]. The $\mathrm{NH}_{3}$ group in configuration [7] is able to return the hydrogen back to the chain and undergo $\mathrm{NH}_{2}$ functional group migration, [12] - [17]. Structures [13] and [15] are all-real frequency complexes with insignificant barriers calculated to be less than $0.01 \mathrm{eV}$ energy difference and as such, the transition states to and from [14] are not indicated in the potential energy surface diagram (Figure S4). The 1,2-hydrogen migration transition of [12] is calculated at $9.07 \mathrm{eV}$, greater than the product sum energy of $\mathbf{R 1}(8.41 \mathrm{eV})$, and for this reason $\mathrm{m} / z=59$ and 44 are less competitive with $m / z=43$ at the dissociation limit energy of $9.13 \mathrm{eV}$ and appear at slightly higher energies. In 
addition, transition state $[16]^{\ddagger}$ at relative energy $8.76 \mathrm{eV}$ is higher in energy still than any stationary point involved in the R1 process to form $m / z=43$.

R2) The formation of a low-energy fragment ion as a result of $\mathrm{NH}_{2}$-loss begins with $\mathrm{H}$-migration to form complex [7], just as in $\mathbf{R} \mathbf{1}$ and $\mathbf{R} \mathbf{2}$ to form $\mathrm{m} / \mathrm{z}=44$ and 59. The H-migration transition [12] at relative energy $9.07 \mathrm{eV}$ is shared with $\mathbf{R 2}$. Unlike in the $\mathbf{R} 2$ channel where the $\mathrm{NH}_{2}$ migrates and then bonds to form [17], the $\mathrm{NH}_{2}$ group dissociates from the complex to form products with energy sum of $8.98 \mathrm{eV}$,

R3) Through our study it is also found that methane-loss to form formamidine $\left(\mathrm{NHCHNH}_{2}{ }^{+}\right)$is theoretically energetically possible, however it is $0.36 \mathrm{eV}$ higher in energy than structure [19] and would involve a multistep process of $\mathrm{NH}_{2}$-group and $\mathrm{CH}_{4}$-group migrations. In addition, the breakdown modeling of the $\mathrm{NH}_{2}$-loss pathway matches more agreeably. It is therefore less likely this fragment forms via $\mathrm{CH}_{4}$-loss and is not included in the potential energy surface diagram.

R4) Potential energy surfaces involving the $\mathrm{C}-\mathrm{C}$ stretching of the higher-energy initial conformers [1] and [3] yield small rotational barriers to structure [5] prior to dissociation. In addition, $m / z$ $=30$ can also form via $\mathrm{C}-\mathrm{C}$ bond cleavage of [7] through a roaming process, whereby the $\mathrm{CH}_{2} \mathrm{NH}_{3}{ }^{+}$and $\mathrm{CHNH}_{2}$ fragments separate and then rotate to return the $\mathrm{H}$ from $\mathrm{CH}_{2} \mathrm{NH}_{3}{ }^{+}$prior to molecular dissociation. Though roaming is a relatively new phenomenon in unimolecular dissociation and has been a topic of recent investigation, ${ }^{4-7}$ it is not expected to play a role in ethylenediamine dissociation in that the product sum energy (calculated at $9.49 \mathrm{eV}$ ) is certainly greater than the barriers witnessed in the various bond-stretching scans.

R5) Various pathways are investigated for the formation of $\mathrm{m} / \mathrm{z}=31$, while only one is believed to be the primary contributor to $\mathbf{R 5}$. The corresponding neutral ligand of mass 29 from the 
dissociation of EN can take one of four forms: methylnitrene $\left(\mathrm{CH}_{3} \mathrm{~N}\right)$, a zwitterion $\left({ }^{+} \mathrm{NH}_{2} \mathrm{CH}^{-}\right.$ ), an aminocarbene $\left(\mathrm{NH}_{2} \mathrm{CH}\right)$, or methylenimine $\left(\mathrm{NHCH}_{2}\right)$. The singlet $\mathrm{CH}_{3} \mathrm{~N}$ is known to be higher in energy, ${ }^{8}$ the zwitterion is calculated with CBS-QB3 to be $1.55 \mathrm{eV}$ higher in energy than $\mathrm{NHCH}_{2}$, and the computational studies of $\mathrm{NH}_{2} \mathrm{CH}$ place it at $35-39 \mathrm{kcal} \mathrm{mol}^{-1}(1.5-1.6$ eV) higher in energy than $\mathrm{NHCH}_{2} \cdot{ }^{8-10}$ The neutral $\mathrm{N}_{2} \mathrm{H}$ structure was also considered, however, the energy is high and the mechanism to form cationic $\mathrm{CH}_{3} \mathrm{CH}_{4}{ }^{+}$at $m / z=31$ was found to be unlikely. Similarly, the calculated energy sum of neutral $\mathrm{HCN}+\mathrm{H}_{2}$ fragments was found to be $0.32 \mathrm{eV}$ higher than $\mathrm{NHCH}_{2}$ and too high to match experimental results. Thus, regardless of the configuration of $m / z=31, \mathbf{R 5}$ must produce the $\mathrm{NHCH}_{2}$ fragment to have energetic agreement with what is observed in the TPEPICO experiment. The pathway leading to the formation of $\mathrm{CH}_{2} \mathrm{NH}_{3}{ }^{+}$, and $\mathrm{NHCH}_{2}$ [23] is enabled by hydrogen-nitrogen interactions and yields a relatively low-energy van der Waals complex, [22], as a result of $\mathrm{CH}_{2} \mathrm{NH}_{2}$ group rotation and $\mathrm{C}-\mathrm{C}$ bond breaking.

Past experiments have contradicted theory ${ }^{11}$ to show the distonic methyleneammonium ion $\left(\mathrm{CH}_{2} \mathrm{NH}_{3}{ }^{+}\right)$to be less stable than its conventional isomer, methanimine $\left(\mathrm{CH}_{3} \mathrm{NH}_{2}\right)^{+}$. However, additional high-level calculations ${ }^{12-13}$ show that the former to be as much as $8 \mathrm{~kJ} \mathrm{~mol}^{-1}(0.08$ eV) more stable. In this exploration using CBS-QB3, an intermediate complex involving C$\mathrm{H}-\mathrm{N}$ interactions leading to the formation of $\mathrm{CH}_{3} \mathrm{NH}_{2}{ }^{+}$is calculated to be $0.09 \mathrm{eV}$ higher in energy than structure [22]. In addition, the resulting energy of $\mathrm{CH}_{3} \mathrm{NH}_{2}{ }^{+}$is also nearly 0.04 $\mathrm{eV}$ higher in energy than the $\mathrm{CH}_{2} \mathrm{NH}_{3}{ }^{+}$, consistent with previous theoretical results obtained. ${ }^{14-15}$ Therefore, through $\mathrm{EN}$ ion dissociation, the $\mathrm{CH}_{2} \mathrm{NH}_{3}{ }^{+}$is preferable to $\mathrm{CH}_{3} \mathrm{NH}_{2}{ }^{+}$. Consecutive dissociation is not likely as it would require substantially more energy. 
R6) The van der Waals product complex [25] undergoes $\mathrm{NH}_{2}$-migration to arrive at a lower-energy product complex [27]. This intermediate is strengthened by intramolecular hydrogen bonding between the two amino groups.

R7) A 1,2-hydrogen shift from a terminal $\mathrm{NH}_{2}$-group to an adjacent carbon is calculated at 10.10 $\mathrm{eV}$ relative energy [33] to yield the $\mathrm{EN}^{+\cdot}$ isomer, methyl-methanediamine ion [34]. This ion in particular is responsible for $\mathbf{R 7}$ and $\mathbf{R 8}$ dissociations. Hydrogen-loss from the central carbon overcomes a small barrier at $9.13 \mathrm{eV}$ to form a resonance-stabilized cationic fragment of $m / z=59$. The loss of a hydrogen at any other site of [34] would require additional energy as it would not lead to a resonance-stabilized product or would break stronger N-H interactions. Indeed, a direct hydrogen abstraction from at the amino site of [32] revealed a CBS-QB3 calculated barrier of $10.31 \mathrm{eV}$ and a product sum energy of $9.69 \mathrm{eV}$, which are the same fragments that would form from the loss of hydrogen at the terminal carbon site of [34]. In addition, the direct loss of a hydrogen from a carbon of either minima [30] or [32] would proceed backward to [5] and yield a CBS-QB3 barrier of $9.56 \mathrm{eV}$, which was much too high in energy to explain the formation of the first $m / z=59$ and much too low to be agreeable with the second.

R8) The bulk of the pathway leading to the second appearance of $m / z=30(\mathbf{R 8})$ is shared with $\mathbf{R 7}$. Rotamers [30] and [32] face low-energy barriers to formation and the hydrogen-transfer barrier at $10.23 \mathrm{eV}$ forms the $\mathrm{EN}^{+\cdot}$ isomer, methyl-methanediamine, at [34]. The bisection of this isomer is uphill and does not possess a barrier to dissociation, once again forming the $\mathrm{CH}_{2} \mathrm{NH}_{2}{ }^{+}$as in $\mathbf{R 4}$, but with a higher-energy neutral fragment radical $\left(\mathrm{CH}_{3} \mathrm{NH}^{-}\right)$at [37]. Though activation energies and product sum energies do not always accurately predict the relative intensities of ions or their appearance order, it is not surprising in the case of $\mathrm{EN}^{+}$ 
dissociation that $\mathbf{R 8}$ channel ion appears at slightly higher energy than $\mathbf{R 7}$. The barrier to formation [35] and the total product energy [36] in $\mathbf{R 7}$ (9.13 and $8.68 \mathrm{eV}$, respectively) are lower than the total product energy [37] of $\mathbf{R 8}(9.77 \mathrm{eV})$, which offers a potential explanation for the later appearance of $\mathrm{m} / \mathrm{z}=30$ despite sharing the same high-energy barrier [33] at 10.23 $\mathrm{eV}$. 


\section{Supplemental Information References}

(1.) Ruscic, B. Active Thermochemical Tables (ATcT) values based on ver. 1.118 of the Thermochemical Network. http://ATcT.anl.gov (accessed March 28, 2016).

(2.) Ruscic, B.; Pinzon, R. E.; Laszewski, G. v.; Kodeboyina, D.; Burcat, A.; Leahy, D.; Montoya, D.; Wagner, A. F. Active Thermochemical Tables: Thermochemistry for the 21st Century. J. Phys. Conf. Ser. 2005, 16, 561-570.

(3.) Ruscic, B.; Pinzon, R. E.; Morton, M. L.; Laszewski, G. v.; Bittner, S.; Nijsure, S. G.; Amin, K. A.; Minkoff, M.; Wagner, A. F. Introduction to Active Thermochemical Tables: Several "Key" Enthalpies of Formation Revisited. J. Phys. Chem. A 2004, 108, 9979-9997.

(4.) Li, H.-K.; Tsai, P.-Y.; Hung, K.-C.; Kasai, T.; Lin, K.-C. Communication: Photodissociation of $\mathrm{CH}_{3} \mathrm{CHO}$ at $308 \mathrm{~nm}$ : Observation of H-roaming, $\mathrm{CH}_{3}$-roaming, and transition state pathways together along the ground state surface. J. Chem. Phys. 2015, 142, 041101.

(5.) Fernando, R.; Dey, A.; Broderick, B. M.; Fu, B.; Homayoon, Z.; Bowman, J. M.; Suits, A. G. Visible/Infrared Dissociation of $\mathrm{NO}_{3}$ : Roaming in the Dark of Roaming on the Ground? J. Phys. Chem. A 2015, 119, 7163-7168.

(6.) Kim, H.-R. Excursion, Roaming and Migration of Hydrogen Atom during Dissociation of Formaldehyde. Bull. Korean Chem. Soc. 2014, 35, 1285-1293.

(7.) Heazlewood, B. R.; Jordan, M. J. T.; Kable, S. H.; Selby, T. M.; Osborn, D. L.; Shepler, B. C.; Braams, B. J.; Bowman, J. M. Roaming is the Dominant Mechanism for Molecular Products in Acetaldehyde Photodissociation. Proceedings of the National Academy of Sciences 2008, 105, $12719-12724$.

(8.) Zhou, J.; Schlegel, H. B. Ab Initio Classical Trajectory Study of the Dissociation of Neutral and Positively Charged Methanimine $\left(\mathrm{CH}_{2} \mathrm{NH}^{\mathrm{n}+} \mathrm{n}=0-2\right)$. J. Phys. Chem. A 2009, 113, 9958-9964. 
(9.) Pople, J. A.; Raghavachari, K.; Frisch, M. J.; Binkley, J. S.; Schleyer, P. V. R. Comprehensive Theoretical Study of Isomers and Rearrangement Barriers of Even-electron Polyatomic Molecules $\mathrm{H}_{\mathrm{m}} \mathrm{ABH}_{\mathrm{n}}(\mathrm{A}, \mathrm{B}=$ Carbon, Nitrogen, Oxygen, and Fluorine). J. Am. Chem. Soc. 1983, 105, 6389-6399.

(10.) Roithova, J.; Schroder, D.; Schwarz, H. Unimolecular Fragmentation of $\mathrm{CH}_{3} \mathrm{NH}_{2}$ : Towards a Mechanistic Description of HCN Formation. E. J. Org. Chem. 2005, 3304-3313.

(11.) Holmes, J. L.; Lossing, F. P.; Terlouq, J. K.; Burgers, P. C. Novel Gas-Phase Ions. The Radical Cations $\left[\mathrm{CH}_{2} \mathrm{XH}\right]^{+}\left(\mathrm{X}=\mathrm{F}, \mathrm{Cl}, \mathrm{Br}, \mathrm{I}, \mathrm{OH}, \mathrm{NH}_{2}, \mathrm{SH}\right)$ and $\left[\mathrm{CH}_{2} \mathrm{CH}_{2} \mathrm{NH}_{3}\right]^{+}$. Can. J. Chem 1983, 61, 2305-2309.

(12.) Yates, B. F.; Nobes, R. H.; Radom, L. The Methyleneammonium Radical Cation $\left(\mathrm{CH}_{2} \mathrm{NH}_{3}{ }^{+}\right)$. Chem. Phys. Lett. 1985, 116, 474-477.

(13.) Sana, M.; Leroy, G.; Hilali, M.; Nguyen, M. T.; Vanquickenborne, L. G. Heats of Formation of Isomeric $\left[\mathrm{C}, \mathrm{H}_{4}, \mathrm{O}\right]^{+},\left[\mathrm{C}, \mathrm{H}_{3}, \mathrm{~N}\right]^{+}$and $\left[\mathrm{C}, \mathrm{H}_{5}, \mathrm{~N}\right]^{+}$Radical Cations. Chem. Phys. Lett. 1992, 190, 551-556.

(14.) Frisch, M. J.; Raghavachari, K.; Pople, J. A.; Bouma, W. J.; Radom, L. Unusual Lowenergy Isomers for Simple Radical Cations. Chem. Phys. Lett. 1983, 75, 323-329.

(15.) Bouma, W. J.; Dawes, J. M.; Radom, L. The Methylamine Radical Cation $\left[\mathrm{CH}_{3} \mathrm{NH}_{2}\right]^{+\bullet}$ and its Stable Isomer the Methylenammonium Radical Cation $\left[\mathrm{CH}_{2} \mathrm{NH}_{3}\right]^{+^{+}}$. J. Mass Spectrom. 1983, 18, 12-15. 